\title{
A Rare 3D Porous Inorganic-Organic Hybrid Polyoxometalate Framework Based on a Cubic Polyoxoniobate-Cupric-Complex Cage with a High Water Vapor Adsorption Capacity
}

Zeng-Kui Zhu, ${ }^{\dagger}$ Ya-Yun Lin, ${ }^{\dagger}$ Li-Dan Lin,${ }^{\dagger}$ Xin-Xiong Li, ${ }^{\dagger}$ Yan-Qiong Sun, ${ }^{\dagger *}$ Shou-Tian Zheng ${ }^{\dagger *}$

†State Key Laboratory of Photocatalysis on Energy and Environment, College of Chemistry, Fuzhou University, Fuzhou, Fujian 350108, China.

This file includes:

$\begin{array}{lr}\text { Section 1: Experimental section } & \text { S1-S2 }\end{array}$

$\begin{array}{lr}\text { Section 2: Additional table } & \text { S2-S3 }\end{array}$

$\begin{array}{ll}\text { Section 3: Additional structural figures and characterizations } & \text { S4-S7 }\end{array}$

$\begin{array}{lr}\text { Section 4: Topology analysis } & \text { S7-S8 }\end{array}$

$\begin{array}{lr}\text { Section 5: References } & \text { S9-S10 }\end{array}$ 


\section{Section 1: Experimental Section}

Materials and General methods: 4-(Tetrazol-5-yl)pyridine ligand is synthesized according to the literature ${ }^{\mathrm{S} 1}$. All other chemicals were commercially purchased and used without further purification. Elemental analyses of $\mathrm{C}, \mathrm{H}$ and $\mathrm{N}$ were carried out with a Vario MICRO elemental analysis. IR spectra were recorded on an Opus Vertex 70 FT-IR infrared spectrophotometer in the range of $400-4000 \mathrm{~cm}^{-1}$. Thermogravimetric analysiswas performed on a Mettler Toledo TGA/SDTA 851e analyzer under an air-flow atmosphere with a heating rate of $10^{\circ} \mathrm{C} / \mathrm{min}$ in the temperature of $30-800^{\circ} \mathrm{C}$. PXRD patterns were obtained by using a Ultima IV diffractometer with $\mathrm{Cu}-K \alpha$ radiation $(\lambda=1.5418 \AA)$ in the range $5-45^{\circ}$. UV-vis adsorption spectra were collected using a PerkinElmer Lambda 35 spectrophotometer to monitor the release process. Fluorescence spectra were measured on an Edinburgh Instrument FS980 TCSPC luminescence spectrometer by using crystalline samples. Single-crystal X-ray diffraction data for $\mathbf{1}$ was collected on a Bruker APEX II diffractometer at $175 \mathrm{~K}$ equipped with a fine focus, $2.0 \mathrm{~kW}$ sealed tube X-ray source (MoK radiation, $\lambda=0.71073 \AA$ ) operating at $50 \mathrm{kV}$ and $30 \mathrm{~mA}$. CCDC-2009018 contains the supplementary crystallographic data for compounds $\mathbf{1}$.

Synthesis of $\mathrm{H}_{20} \mathrm{Cu}(\mathrm{en})\left[\mathrm{Cu}(\mathrm{en})_{2}\right]_{11}\left\{\left\{\left[\mathrm{Cu}(\mathrm{en})_{2}\right] @\left\{\left[\mathrm{Cu}_{2}(\mathrm{en})_{2}(\operatorname{trz})_{2}\right]_{6}\left(\mathrm{Nb}_{68} \mathrm{O}_{188}\right)\right\}[4-\mathrm{Tzp}]\right\}_{2}\right.$ .22en.130H $\mathrm{H}_{2} \mathrm{O}$ (1): A mixture of $\mathrm{K}_{7} \mathrm{HNb}_{6} \mathrm{O}_{19} \cdot 13 \mathrm{H}_{2} \mathrm{O}(0.274 \mathrm{~g}, 0.20 \mathrm{mmol}), \mathrm{Cu}(\mathrm{OAc})_{2} \cdot \mathrm{H}_{2} \mathrm{O}(0.30 \mathrm{~g}$, $1.5 \mathrm{mmol}), \mathrm{Na}_{2} \mathrm{CO}_{3}(0.105 \mathrm{~g}, 1 \mathrm{mmol}), \mathrm{Li}_{2} \mathrm{~B}_{4} \mathrm{O}_{7}(0.083 \mathrm{~g}, 0.5 \mathrm{mmol}), 1,2,4$-Triazole $(0.138 \mathrm{~g}, 2$ mmol), 4-(Tetrazol-5-yl)pyridine $(0.147 \mathrm{~g}, 1 \mathrm{mmol})$ and $90 \mu \mathrm{L}$ en was mixed in $8 \mathrm{~mL}$ deionized water. After stirred 1 hour, the resulting mixture was sealed in a glass vial $(20 \mathrm{ml})$ and heated at 90 ${ }^{\circ} \mathrm{C}$ for 3 days. After slow cooling down to room temperature for two days, blue purple rod crystals were obtained. Yield: about $40 \mathrm{mg}$ ( $15 \%$ based on $\mathrm{Nb})$. The $\mathrm{pH}$ values before and after the reaction are 7.9 and 8.5, respectively. Elemental analysis calcd (found\%) for $\mathrm{H}_{910} \mathrm{C}_{204} \mathrm{~N}_{226} \mathrm{Cu}_{38} \mathrm{Nb}_{136} \mathrm{O}_{505}$ : C,8.26; H, 3.09; N, 10.67; Cu, 8.14; Nb, 42.60. Found: C, 8.33; H, 3.33; N, 10.87; Cu, 8.02; Nb, 42.31. IR (solid ATR, $v / \mathrm{cm}^{-1}$ ): 3231(vs), 3101(s), 1589(s), 1412(s), 1294(w), 1191(w), 1104(w), 1041(vs), 991(m), 925(m), 840(m), 772(s), 616(s), 469(m).

\section{Synthesis discussion}

In order to construct a PONb-based framework material, we did a further analysis of the cubic cage 1a. We believe that there are still uncoordinated metal sites on the $\mathrm{Cu}^{2+}$ ion in the $\mathrm{Cu}_{2}$ motifs. So we tried to add different types of ligands into the PONb-metal-complex cage system, such as trimesic acid, p-phthalic acid, 3,5-pyridinedicarboxylic acid, 4,4'-bipyridine, 4,4'-bi-4H-1,2,4triazole, 4-(Tetrazol-5-yl)pyridine(4-Tzp). Unfortunately, only 4-Tzp ligands could be linked with $\mathrm{Cu}_{2}$ motifs to form a novel cubic cage based open framework. Analyzing the reason, we found that compared to carboxylic acid ligands, the 4-Tzp ligand deprotonation process does not cause a drastic change in the $\mathrm{pH}$ of the solution, and could maintain the $\mathrm{pH}$ similar to other PONb- metal-complex cage system before and after the reaction. Compared with 4,4'-bipyridine, the tetrazole moiety in the 4-Tzp ligand has a stronger coordination ability with $\mathrm{Cu}^{2+}$ ions. After the tetrazole moiety was coordinated with cage $1 \mathbf{a}$, the pyridine $\mathrm{N}$ moiety could continue to react with $\mathrm{Cu}_{2}$ moiety in the adjacent cage 1a. And compared with 4,4'-bi-4H-1,2,4-triazole, the 4,4'-bi-4H-1,2,4-triazole ligand is not stable under strong alkaline conditions and will decomposed into 1,2,4-triazole. We can synthesize the compound that have been reported by our group ${ }^{2}$ when only add 4,4'-bi-4H-1,2,4triazole as the ligand. Therefore, we believe that the 4,4'-bi-4H-1,2,4-triazole ligand is not stable 
under strong alkaline conditions.

Water adsorption measurement: As-synthesized sample 1 was exchanged with excessive ethanol 9 times for 3 days. The solvent exchanged sample was then degassed at $80^{\circ} \mathrm{C}$ for $12 \mathrm{~h}$ under high dynamic vacuum. Water sorption isotherm was recorded at $298 \mathrm{~K}$ on a micromeritics 3 flex Adsorption Analyzer.

Ionic conductivity experiments: Ac impedance measurements were carried out with a SI 1260 IMPEDANCE/GAINPHASE analyzer over the frequency range from $0.1 \mathrm{~Hz}$ to $10 \mathrm{MHz}$ with an applied voltage of $50 \mathrm{mV}$. The relative humidity was controlled by a STIKCorp CIHI-150BS3 incubator. The samples were pressed to form a cylindrical pellet of crystalline powder sample $(\sim 1$ $\mathrm{mm}$ thickness $\times 5 \mathrm{~mm} \phi$ ) coated with C-pressed electrodes. Two silver electrodes were attached to both sides of pellet to form four end terminals (quasi-four-probe method). The bulk conductivity was estimated by semicircle fittings of Nyquist plots.

\section{Section 2: Additional table}

Table S1 X-ray crystallographic data for $\mathbf{1}$

\begin{tabular}{|c|c|}
\hline & 1 \\
\hline Empirical formula & $\mathrm{H}_{910} \mathrm{C}_{204} \mathrm{~N}_{226} \mathrm{Cu}_{38} \mathrm{Nb}_{136} \mathrm{O}_{505}$ \\
\hline Formula weight & 29662.64 \\
\hline Crystal system & Monoclinic \\
\hline space group & $P 2_{1} / c$ \\
\hline$a(\AA)$ & $32.836(4)$ \\
\hline$b(\AA)$ & $37.156(4)$ \\
\hline$c(\AA)$ & 39.940(4) \\
\hline$\beta\left(^{\circ}\right)$ & $92.0735(15)$ \\
\hline$V\left(\AA^{3}\right)$ & $48697(9)$ \\
\hline $\mathrm{Z}$ & 2 \\
\hline $\mathrm{F}(000)$ & 25724 \\
\hline pcalcd $\left(\mathrm{g} \cdot \mathrm{cm}^{-3}\right)$ & 1.809 \\
\hline Temperature (K) & 175 \\
\hline Refl. Collected & 130788 \\
\hline Independent relf. & 67832 \\
\hline Parameters & 4054 \\
\hline GOF on $\mathrm{F}^{2}$ & 1.001 \\
\hline
\end{tabular}


Final $R$ indices $(I=2 \sigma(I))$

$$
R_{l}=0.0946 \quad w R_{2}=0.2372
$$

$R$ indices (all data)

$\mathrm{R}_{1}=\sum|| \mathrm{F}_{\mathrm{o}}|-| \mathrm{F}_{\mathrm{c}}|| / \sum\left|\mathrm{F}_{\mathrm{o}}\right| \cdot \mathrm{wR}_{2}=\left[\sum \mathrm{w}\left(\mathrm{F}_{\mathrm{o}}^{2}-\mathrm{F}_{\mathrm{c}}^{2}\right)^{2} / \sum \mathrm{w}\left(\mathrm{F}_{\mathrm{o}}^{2}\right)^{2}\right]^{1 / 2} ; \mathrm{w}=1 /\left[\sigma^{2}\left(\mathrm{~F}_{\mathrm{o}}^{2}\right)+(\mathrm{xP})^{2}+\mathrm{yP}\right], \mathrm{P}=\left(\mathrm{F}_{\mathrm{o}}^{2}+2 \mathrm{~F}_{\mathrm{c}}^{2}\right) / 3$, where $\mathrm{x}=0.1550, \mathrm{y}=0$ for $\mathbf{1}$

Table S2. A summary of known vapour adsorption capacities of polyoxometalate materials.

\begin{tabular}{|c|c|c|}
\hline Compounds ${ }^{[\mathrm{a}]}$ & Amount $\left(\mathrm{cm}^{3} \mathrm{~g}^{-1}\right)$ & ref \\
\hline 1 & 219 & This work \\
\hline$\left[\mathrm{Cu}(\mathrm{en})_{2}\right]_{6}\left\{\left[\mathrm{Cu}(\mathrm{en})_{2}\right] @\left\{\left[\mathrm{Cu}_{2}(\operatorname{trz})_{2}(\mathrm{en})_{2}\right]_{6}\left[\mathrm{H}_{10} \mathrm{Nb}_{68} \mathrm{O}_{188}\right]\right\}\right\}$ & 224 & $\mathrm{~S} 2$ \\
\hline $\mathrm{K}_{4} @\left\{\left[\mathrm{Cu}_{29}(\mathrm{OH})_{7}\left(\mathrm{H}_{2} \mathrm{O}\right)_{2}(\mathrm{en})_{8}(\operatorname{trz})_{21}\right]\left[\mathrm{Nb}_{24} \mathrm{O}_{67}(\mathrm{OH})_{2}\left(\mathrm{H}_{2} \mathrm{O}\right)_{3}\right]_{4}\right\}$ & 193 & $\mathrm{~S} 2$ \\
\hline$\left[\mathrm{Cu}(\mathrm{en})_{2}\right] @\left\{\left[\mathrm{Cu}_{2}(\mathrm{en})_{2}(\operatorname{trz})_{2}\right]_{6}\left(\mathrm{Nb}_{68} \mathrm{O}_{188}\right)\right\}$ & 188 & $\mathrm{~S} 2$ \\
\hline$\left[\mathrm{Zn}_{12}(\operatorname{trz})_{20}\right]\left[\mathrm{SiW}_{12} \mathrm{O}_{40}\right] \cdot 11 \mathrm{H}_{2} \mathrm{O}$ & 150 & $\mathrm{~S} 3$ \\
\hline $\mathrm{K}_{3}\left[\mathrm{Cr}_{3} \mathrm{O}(\mathrm{OOCH})_{6}\left(\mathrm{H}_{2} \mathrm{O}\right)_{3}\right]\left[\mathrm{R}-\mathrm{SiW}_{12} \mathrm{O}_{40}\right]$ & 130 & $\mathrm{~S} 4$ \\
\hline $\mathrm{Cu}_{6}(\mathrm{Trz})_{10}\left(\mathrm{H}_{2} \mathrm{O}\right)_{4}\left[\mathrm{H}_{2} \mathrm{SiW}_{12} \mathrm{O}_{40}\right] \cdot 8 \mathrm{H}_{2} \mathrm{O}$ & 118 & S5 \\
\hline$\left[\mathrm{Cu}_{4}(\mathrm{dpdo})_{12}\right]\left[\mathrm{H}\left(\mathrm{H}_{2} \mathrm{O}\right)_{27}\left(\mathrm{CH}_{3} \mathrm{CN}\right)_{12}\right]\left[\mathrm{PW}_{12} \mathrm{O}_{40}\right]_{3}$ & 65.1 & S6 \\
\hline $\mathrm{K}_{2}\left[\mathrm{Cr}_{3} \mathrm{O}(\mathrm{OOCH})_{6}(\text { mepy })_{3}\right]_{2}\left[\alpha-\mathrm{PMo}_{12} \mathrm{O}_{40}\right] \cdot 5 \mathrm{H}_{2} \mathrm{O}$ & 56.8 & S7 \\
\hline $\mathrm{H}_{14}\left[\mathrm{Na}_{6}\left(\mathrm{H}_{2} \mathrm{O}\right)_{12}\right]_{4}\left[\mathrm{~K}_{42} \mathrm{Ge}_{8} \mathrm{~W}_{72} \mathrm{O}_{272}\left(\mathrm{H}_{2} \mathrm{O}\right)_{60}\right] \cdot$ solvent & 52 & S8 \\
\hline$\left[\mathrm{Cu}_{3}(\mathrm{~L})_{2}\left(\mathrm{H}_{2} \mathrm{O}\right)_{4}\right]\left[\mathrm{Cu}(\mathrm{dmf})_{4}\left(\mathrm{SiW}_{12} \mathrm{O}_{40}\right)\right] \cdot 9 \mathrm{H}_{2} \mathrm{O}$ & 51.7 & S9 \\
\hline $\left.\mathrm{H}\left[\mathrm{Ni}(\mathrm{Hbpdc})\left(\mathrm{H}_{2} \mathrm{O}\right)_{2}\right]_{2}\left[\mathrm{PW}_{12} \mathrm{O}_{40}\right] \cdot 8 \mathrm{H}_{2} \mathrm{O}\right\}$ & 31 & S10 \\
\hline$\left[\mathrm{Co}(\mathrm{pn})_{3}\right]_{4}\left[\mathrm{PNb}_{12} \mathrm{O}_{40}(\mathrm{VO})_{6}\right][\mathrm{OH}]_{5} \cdot 20 \mathrm{H}_{2} \mathrm{O}$ & 19.72 & S11 \\
\hline$(\mathrm{DODA})_{23}\left[\mathrm{Mo}_{154} \mathrm{O}_{462} \mathrm{H}_{5}\right] \cdot 70 \mathrm{H}_{2} \mathrm{O}$ & 16.6 & $\mathrm{~S} 12$ \\
\hline $\mathrm{Cs}_{3.6} \mathrm{~K}_{0.4}\left[\mathrm{PW}_{11} \mathrm{O}_{39}(\mathrm{Sn}-\mathrm{OH})\right] \cdot 8 \mathrm{H}_{2} \mathrm{O}$ & 0.31 & S13 \\
\hline $\mathrm{K}_{2}\left[\mathrm{Cr}_{3} \mathrm{O}(\mathrm{OOCH})_{6}(\text { mepy })_{3}\right]_{2}\left[\mathrm{a}-\mathrm{SiW}_{12} \mathrm{O}_{40}\right] \cdot 2 \mathrm{H}_{2} \mathrm{O} \cdot \mathrm{CH}_{3} \mathrm{OH}$ & 0.03 & $\mathrm{~S} 14$ \\
\hline $\mathrm{Cs}_{2}\left[\mathrm{Cr}_{3} \mathrm{O}\left(\mathrm{OOCC}_{2} \mathrm{H}_{5}\right)_{6}\left(\mathrm{H}_{2} \mathrm{O}\right)_{3}\right]_{2}\left[\mathrm{R}-\mathrm{SiW}_{12} \mathrm{O}_{40}\right] \cdot 4 \mathrm{H}_{2} \mathrm{O}$ & 0.022 & $\mathrm{~S} 15, \mathrm{~S} 16$ \\
\hline $\mathrm{Cs}_{3} \mathrm{H}_{0.3}\left[\mathrm{SiW}_{12} \mathrm{O}_{40}\right]_{0.83} \cdot 3 \mathrm{H}_{2} \mathrm{O}$ & 0.020 & S17 \\
\hline
\end{tabular}

[a] Trz: 1,2,4-triazole; dpdo: 4,4'-bipyridine-N,N'-dioxide; mepy: 4-methylpyridine; L: N,N-bis[(2hydroxy-3-methoxyphenyl) methylidene] hydrazine hydrate ; dmf N,N-Dimethylformamide; $\mathrm{H}_{2}$ bpdc : 2,2'-bipyridyl-3,3'-dicarboxylicacid ； pn: 1,2-diaminopropane ； DODA: dimethyldioctadecylammonium. 
Section 3: Additional structural figures and characterizations

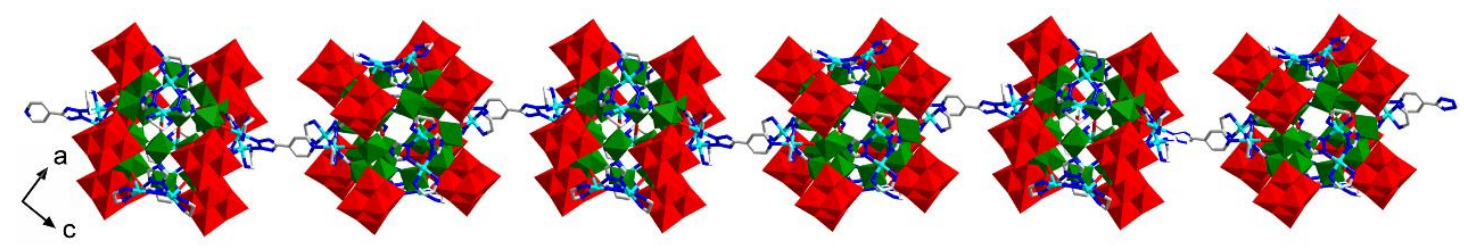

Figure S1. View of 1D pillar chain structure in compound 1 running along [101] direction.

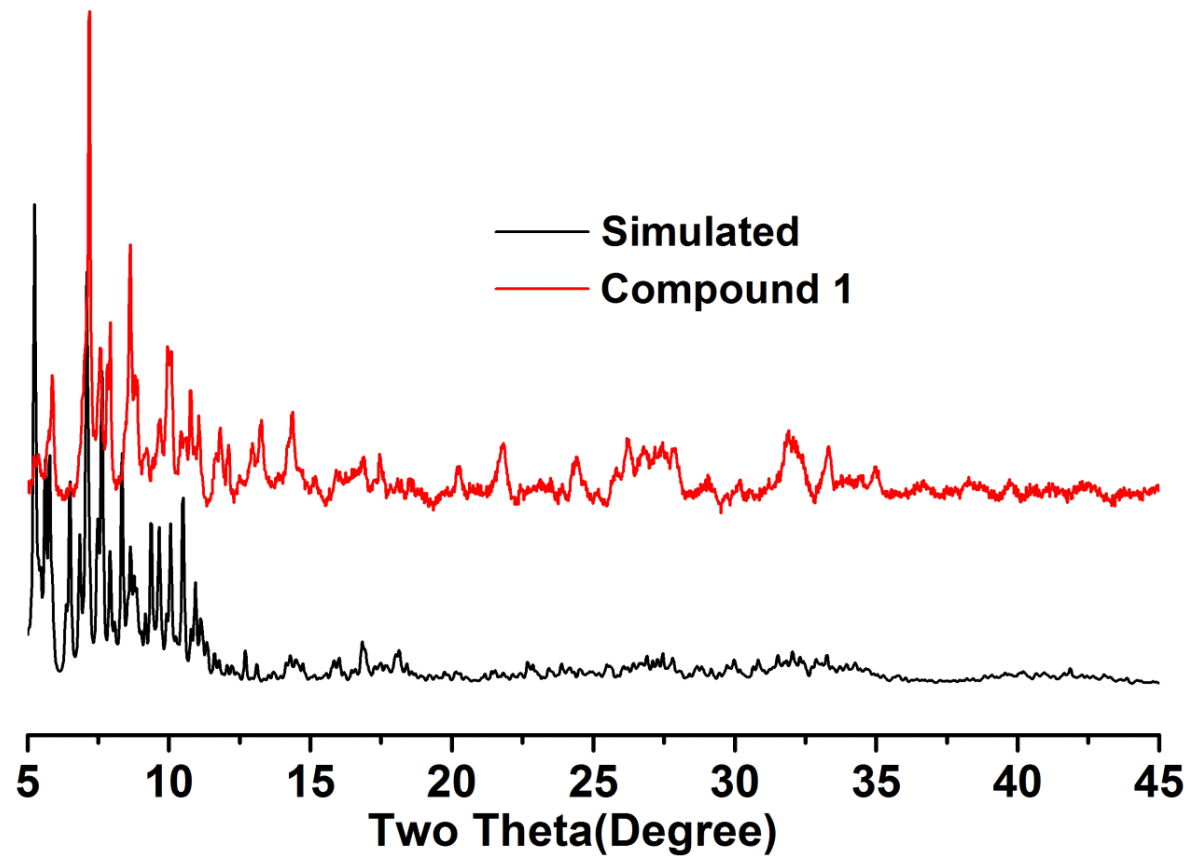

Figure S2. Simulated and experimental PXRD patterns of $\mathbf{1}$.

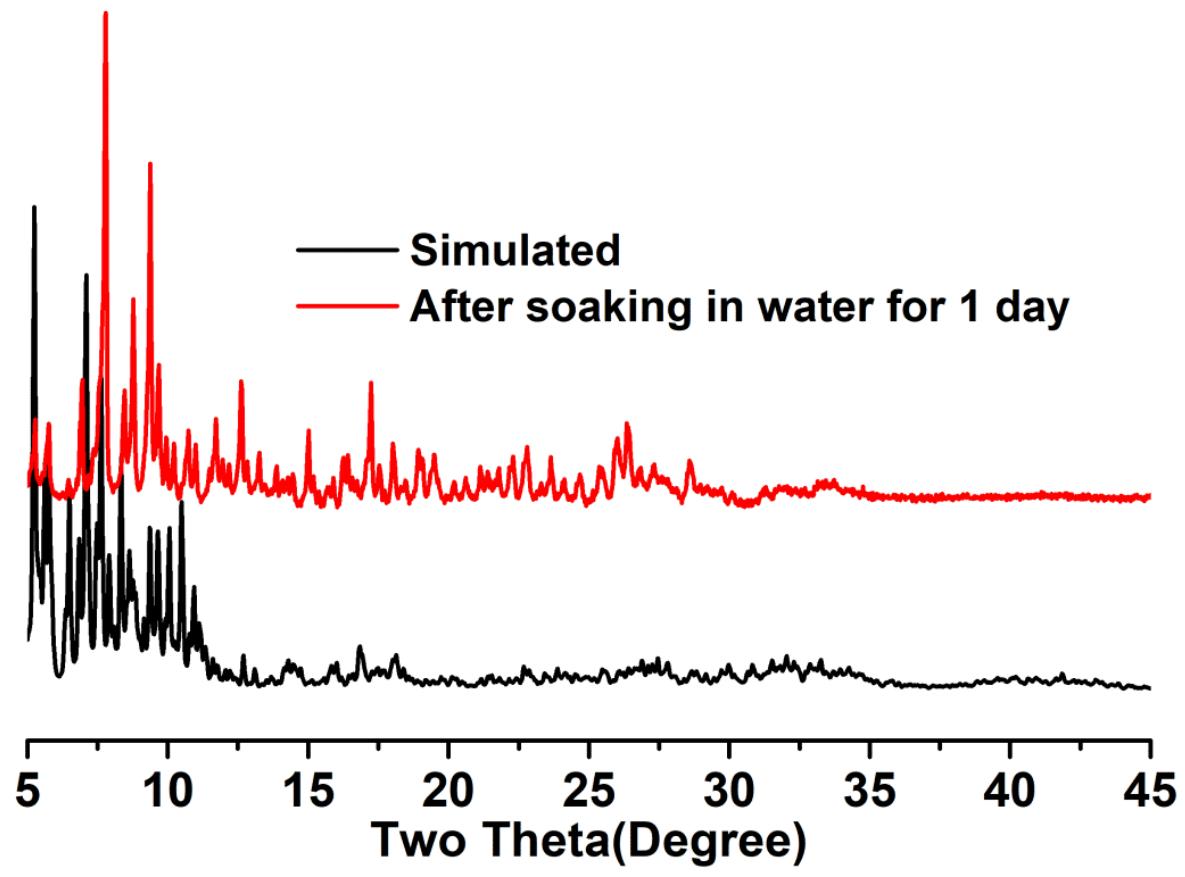

Figure S3. Simulated PXRD pattern and experimental PXRD pattern of $\mathbf{1}$ soaked in water for one day. 

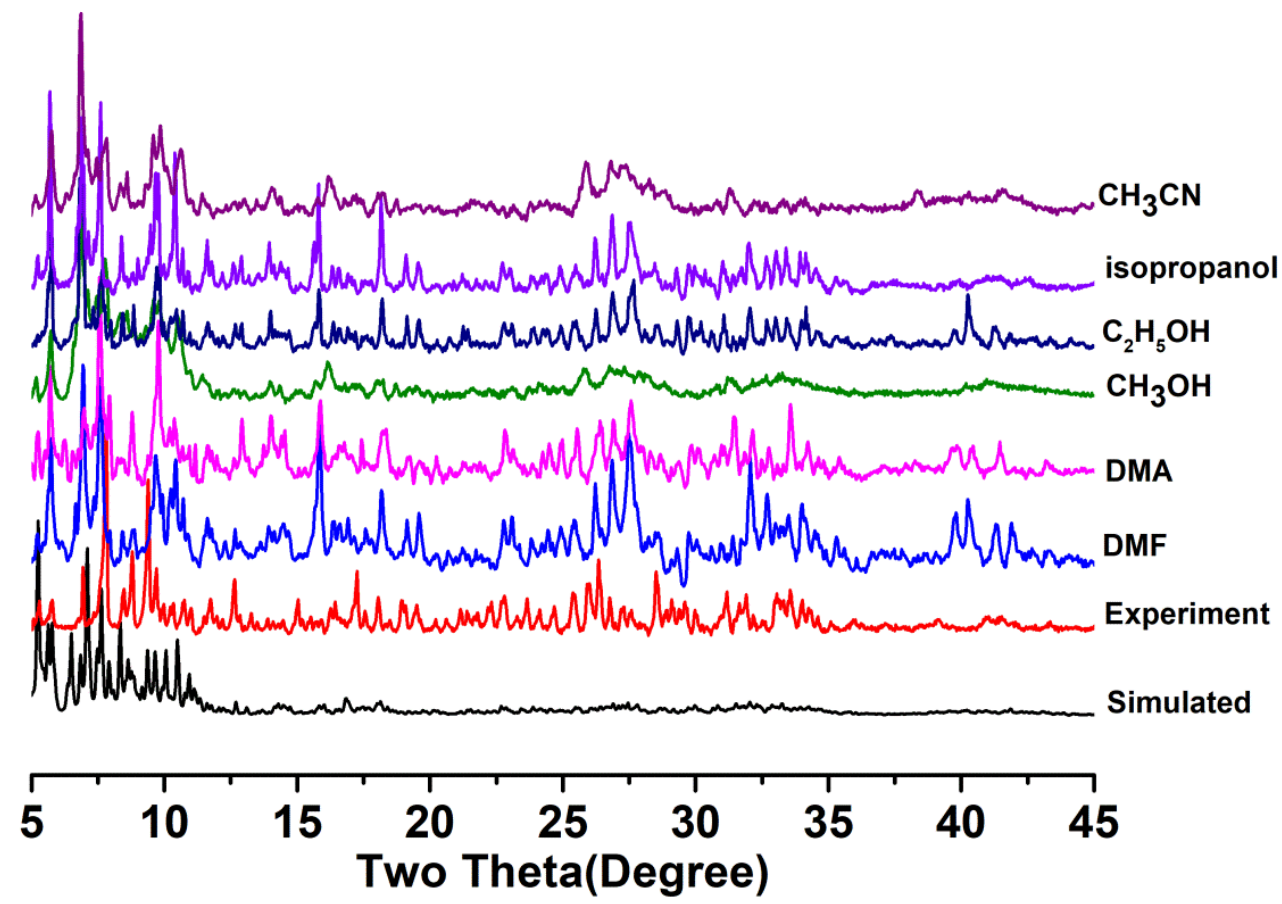

Figure S4. Simulated PXRD pattern and experimental PXRD pattern of $\mathbf{1}$ soaked in different organic solvents for one day.

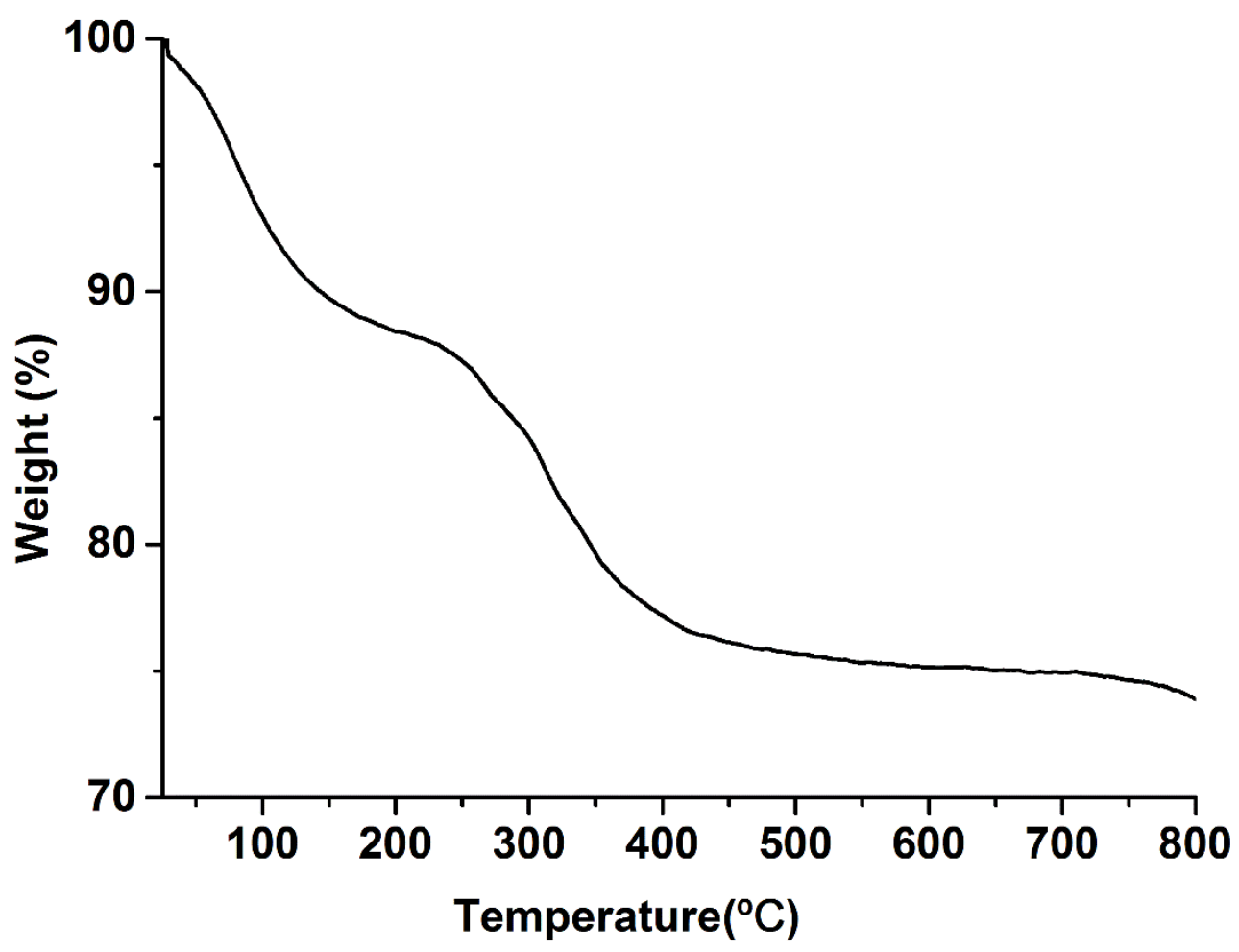

Figure S5. TGA curve of compound $\mathbf{1}$. 


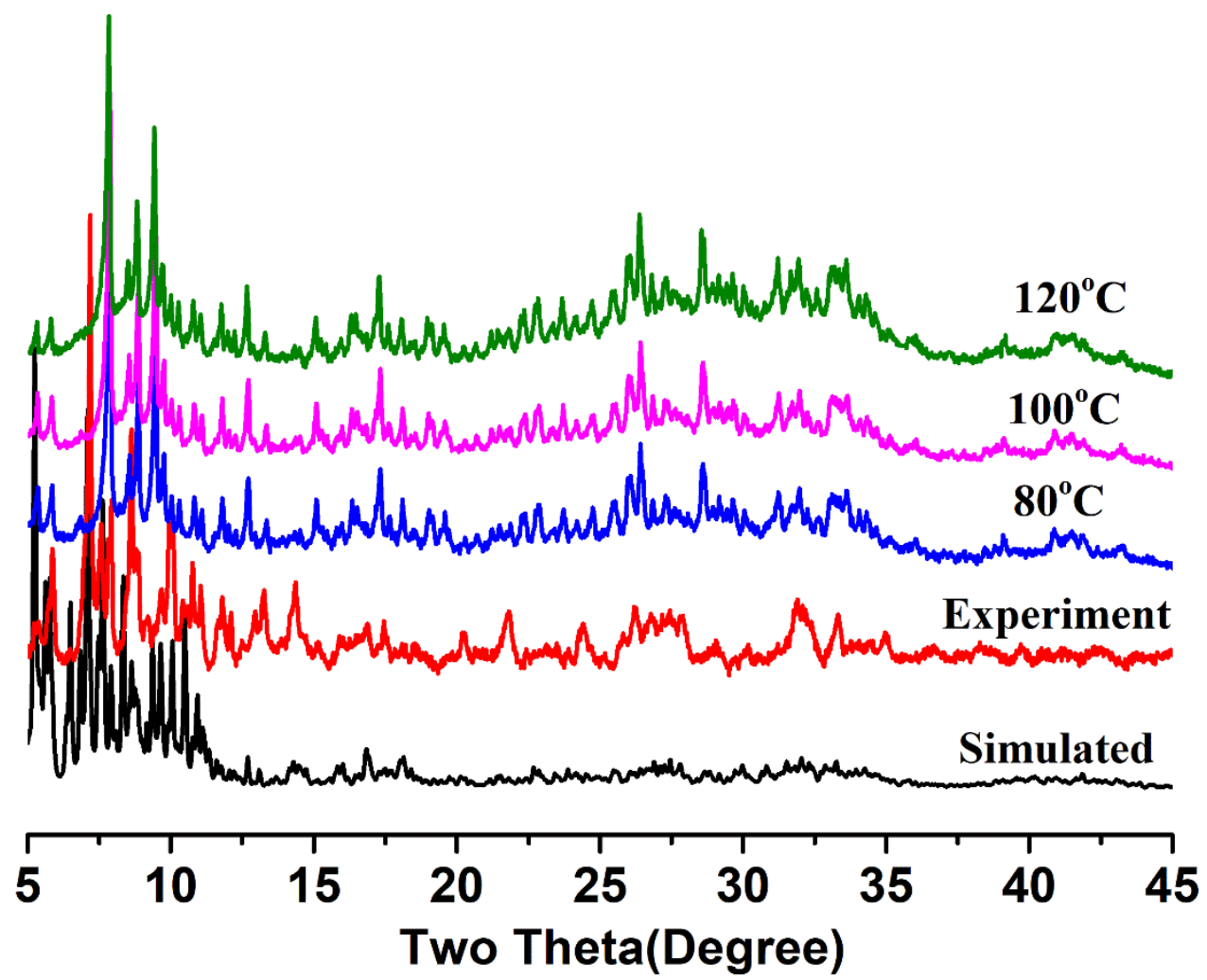

Figure S6. Simulated PXRD pattern and experimental PXRD patterns of $\mathbf{1}$ in different temperature.
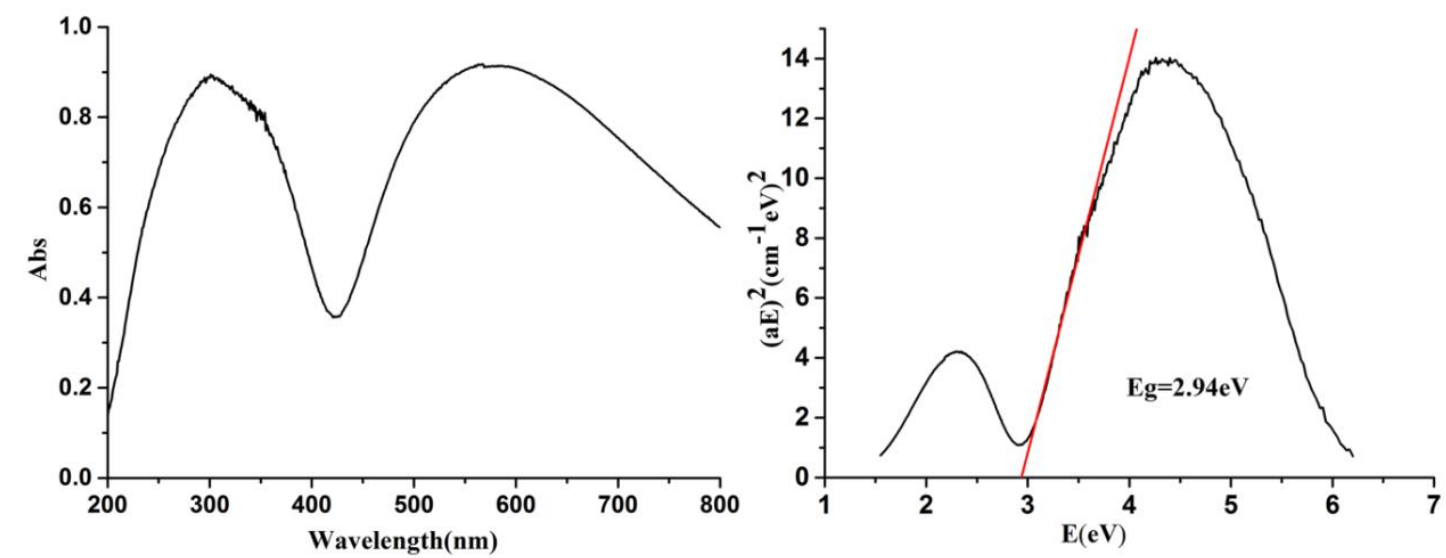

Figure S7. Solid-state diffuse reflectance UV-vis spectrum of $\mathbf{1 .}$ 


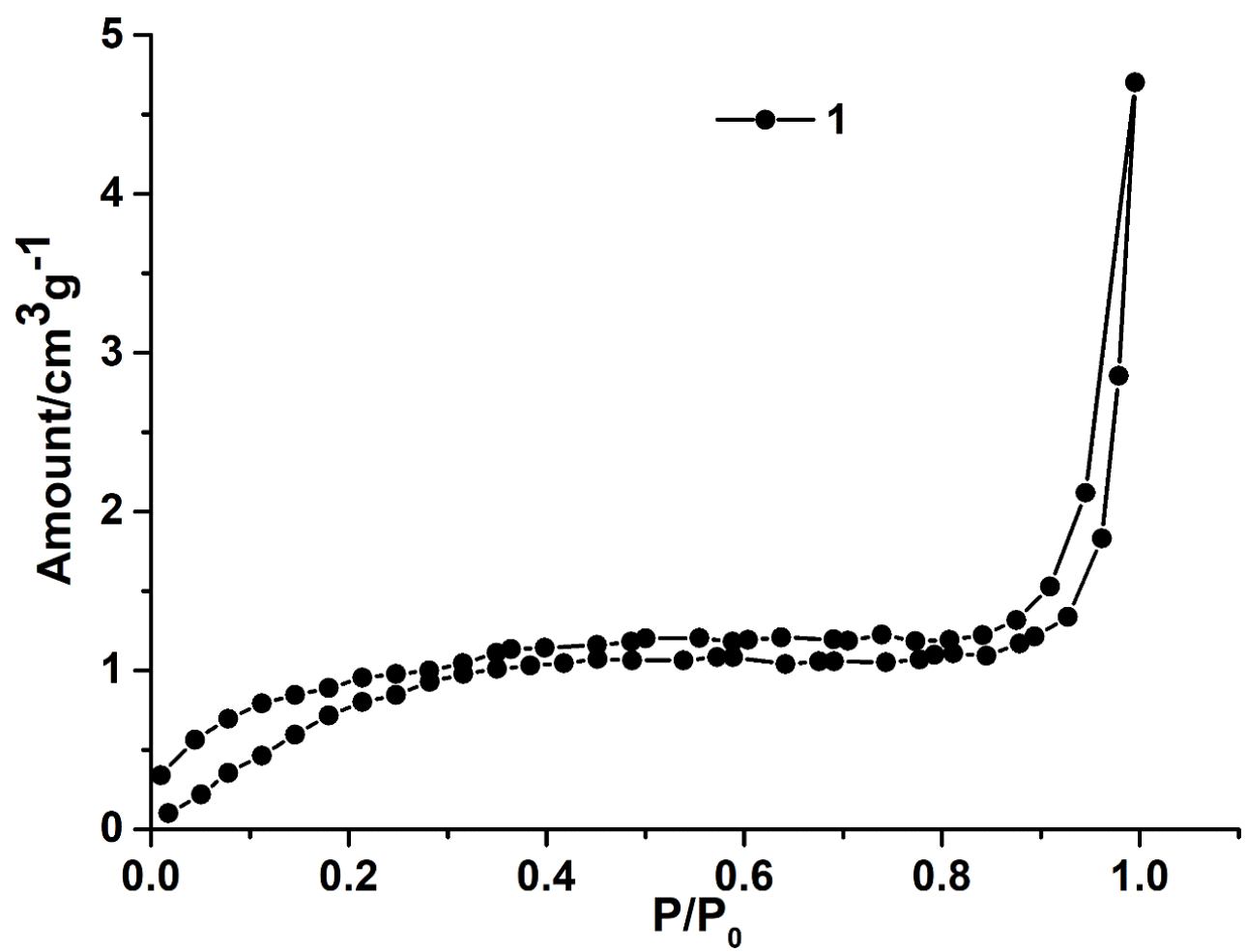

Figure S8. $\mathrm{N}_{2}$ adsorption curve of compound $\mathbf{1}$

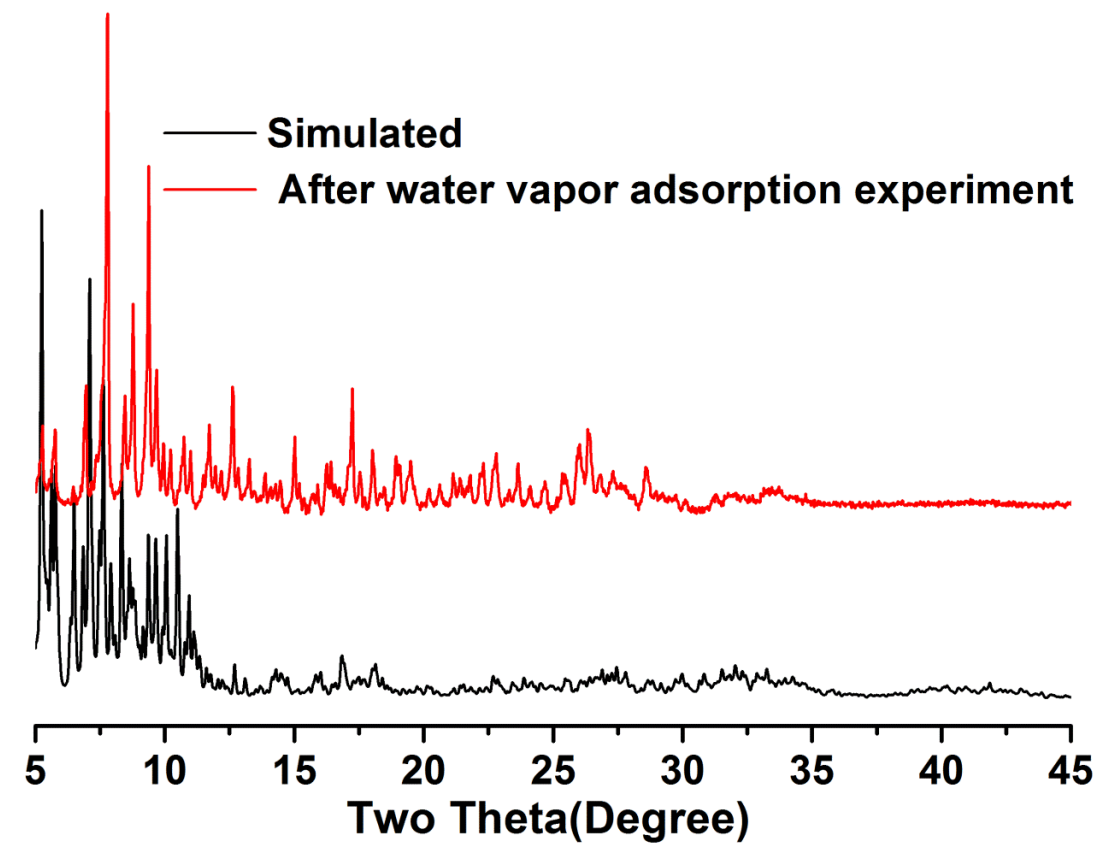

Figure S9. Simulated PXRD pattern and experimental PXRD pattern of $\mathbf{1}$ after water vapour adsorption experiment. 


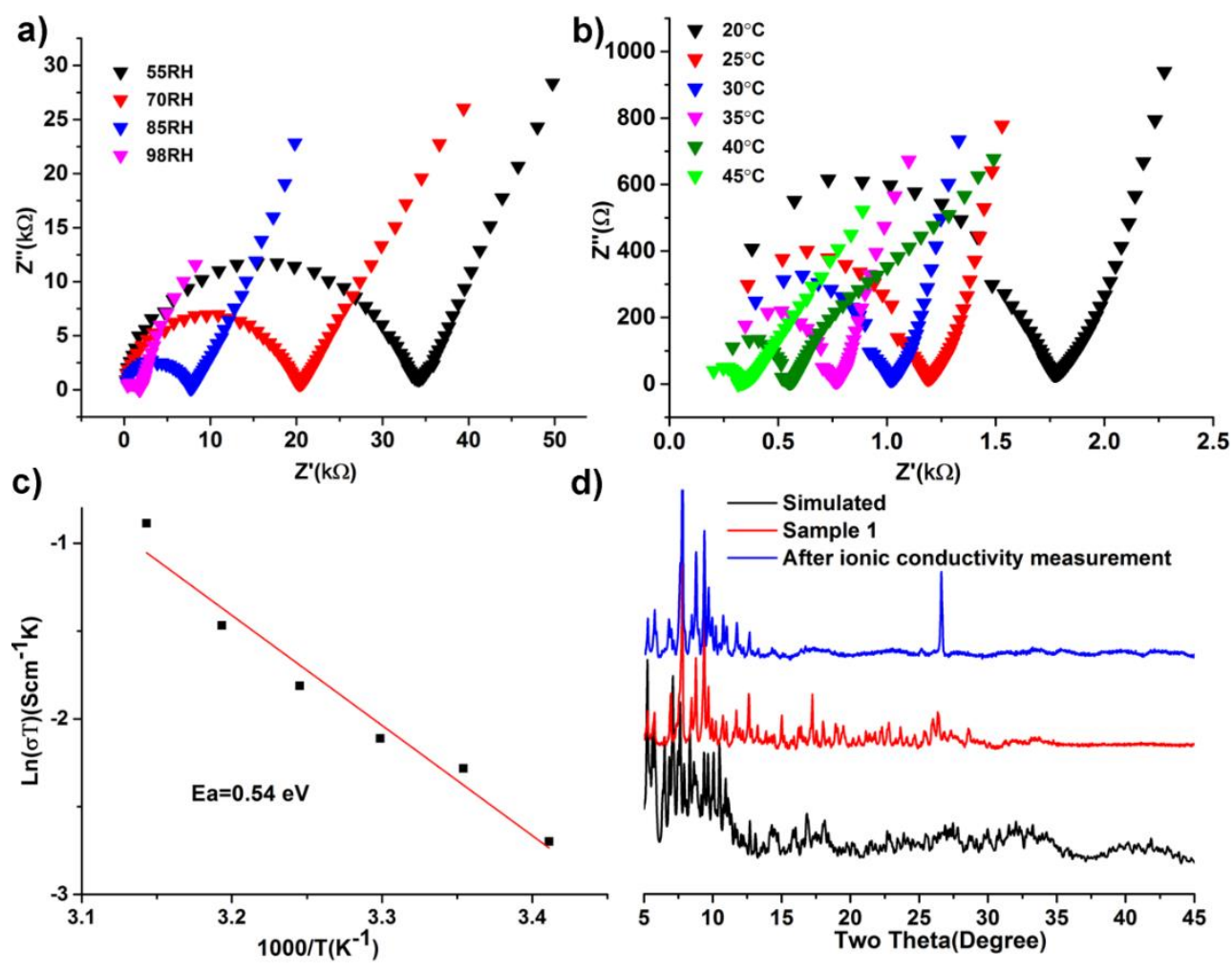

Figure S10. a) Nyquist plot for $\mathbf{1}$ under different RHs and at $20^{\circ} \mathrm{C}$; b) Nyquist plot for $\mathbf{1}$ under different temperatures with $98 \%$ RH; c) Arrhenius plots of the ionic conductivity of $\mathbf{1}$; d) PXRD pattern of 1 after ionic conductivity measurement.

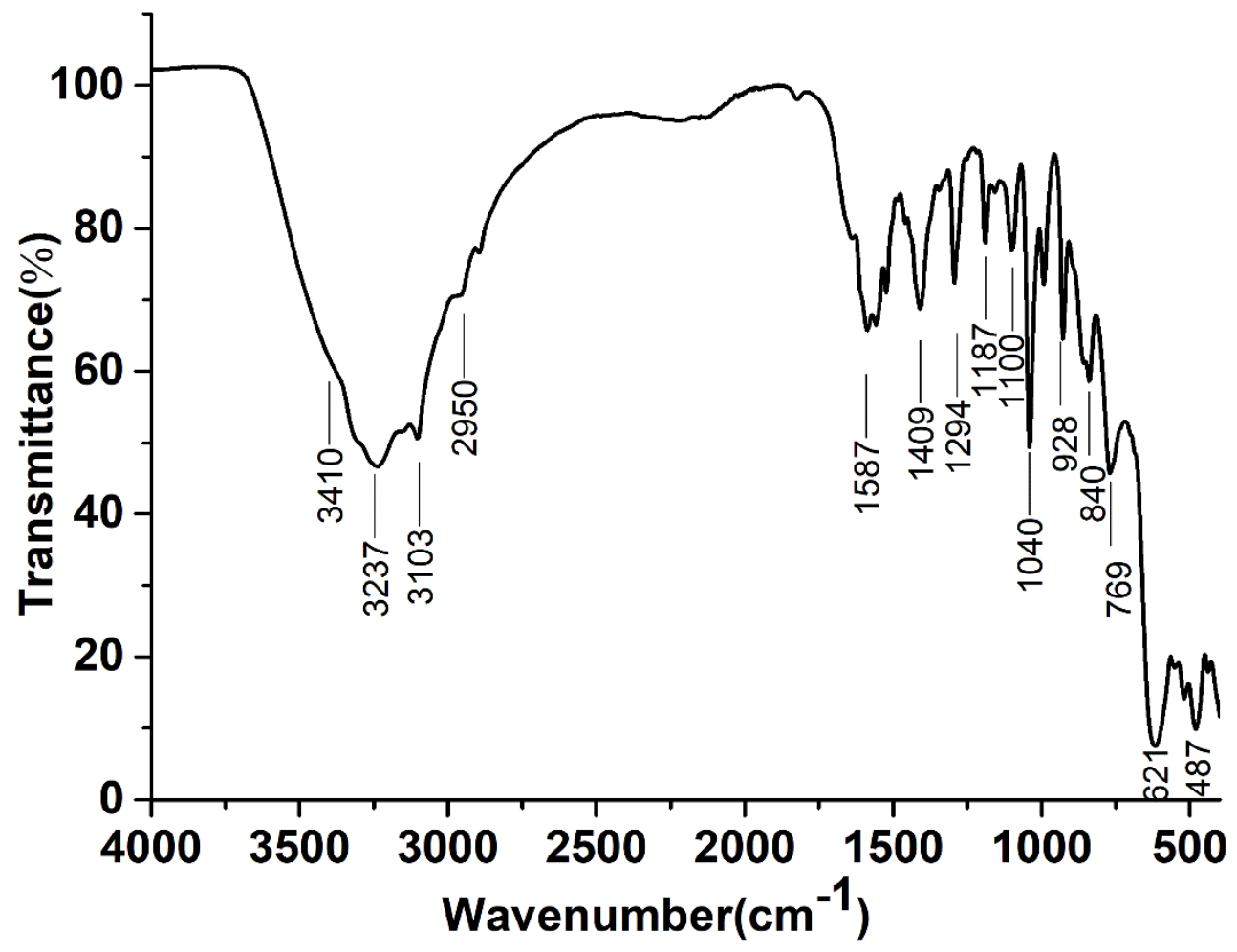

Figure S11. IR spectrum of $\mathbf{1}$.

As shown in Figure $\mathrm{S} 11$, the $\mathrm{CH}_{2}$ and $\mathrm{NH}_{2}$ stretching bands are observed at $\tilde{\mathrm{v}}=$ 2900 to $3200 \mathrm{~cm}^{-1}$, and their bending bands are observed at $v^{\sim}=1100$ to $1600 \mathrm{~cm}^{-1}$. 
The characteristic peaks at $\tilde{\mathrm{v}}=1040,928,840,769,621$, and $487 \mathrm{~cm}^{-1}$ are assigned to the $v\left(\mathrm{Nb}-\mathrm{O}_{\mathrm{t}}\right)$ and $v\left(\mathrm{Nb}-\mathrm{O}_{\mathrm{b}}-\mathrm{M}\right)$ stretches $(\mathrm{M}=\mathrm{Nb}, \mathrm{Cu})$. The peak at $\tilde{\mathrm{v}}=3410 \mathrm{~cm}^{-1}$ is assigned to the water molecules.

As shown in Figure S11, in the high wavenumber region, the strong and wide peaks between $3000 \mathrm{~cm}^{-1}-3500 \mathrm{~cm}^{-1}$ correspond to the $\mathrm{O}-\mathrm{H}$ stretching vibration of coordination water molecules. The peaks appear at $3247 \mathrm{~cm}^{-1}$ and $3100 \mathrm{~cm}^{-1}$ are assigned to the stretching vibration of $\mathrm{C}-\mathrm{H}$ and N-H, respectively. And further the peaks at $1591 \mathrm{~cm}^{-1}$ and $1525 \mathrm{~cm}^{-1}$ are corresponding to the bending vibration of $\mathrm{C}-\mathrm{H}$ and $\mathrm{N}-\mathrm{H}$, respectively. The peak at $1041 \mathrm{~cm}^{-1}$ is assigned to the stretching vibration of $\mathrm{Cu}-\mathrm{O}$. The peaks appearing in the range of $400-1000 \mathrm{~cm}^{-1}$ can be attributed to the characteristic absorption peaks of $\mathrm{Nb}-\mathrm{O}$, of which $843 \mathrm{~cm}^{-1}$ is the stretching vibration peak of $\mathrm{Nb}=\mathrm{Ot}$, and 621 and $483 \mathrm{~cm}^{-1}$ belong to bridging $\mathrm{Nb}-\mathrm{Ob}-\mathrm{Nb}$ stretching vibration.

\section{Section 4: Topology analysis}

Topology for $\mathrm{Cu} 1$

Atom $\mathrm{Cu} 1$ links by bridge ligands and has

Common vertex with

$\mathrm{R}(\mathrm{A}-\mathrm{A})$

$\begin{array}{lllrrrr}\mathrm{Cu} 2 & 1.0000 & 1.0000 & 0.0000 & \left(\begin{array}{lll}1 & 0 & 0\end{array}\right) & 24.793 \mathrm{~A} & 1 \\ \mathrm{Cu} 2 & 0.0000 & 0.0000 & 0.0000 & \left(\begin{array}{lll}0 & -1 & 0\end{array}\right) & 24.793 \mathrm{~A} & 1 \\ \mathrm{Cu} 2 & 0.0000 & 0.5000 & -0.5000 & \left(\begin{array}{ll}0 & 0-1\end{array}\right) & 25.389 \mathrm{~A} & 1 \\ \mathrm{Cu} 2 & 1.0000 & 0.5000 & 0.5000 & \left(\begin{array}{lll}1 & 0 & 0\end{array}\right) & 25.389 \mathrm{~A} & 1\end{array}$

Topology for $\mathrm{Cu} 2$

Atom $\mathrm{Cu} 2$ links by bridge ligands and has

Common vertex with

$\mathrm{R}(\mathrm{A}-\mathrm{A})$

$\begin{array}{rrrrrrr}\mathrm{Cu} 1 & 0.5000 & 0.0000 & 0.5000 & \left(\begin{array}{lll}1 & -1 & 0\end{array}\right) & 24.793 \mathrm{~A} & 1 \\ \mathrm{Cu} 1 & -0.5000 & 1.0000 & 0.5000 & \left(\begin{array}{lll}0 & 0 & 0\end{array}\right) & 24.793 \mathrm{~A} & 1 \\ \mathrm{Cu} 1 & -0.5000 & 0.5000 & 0.0000 & \left(\begin{array}{lll}-1 & 0 & 0\end{array}\right) & 25.389 \mathrm{~A} & 1 \\ \mathrm{Cu} 1 & 0.5000 & 0.5000 & 1.0000 & \left(\begin{array}{lll}0 & 0 & 1\end{array}\right) & 25.389 \mathrm{~A} & 1\end{array}$

Structural group analysis

Structural group No 1

Structure consists of 3D framework with $\mathrm{Cu}$

Coordination sequences 
$\begin{array}{lllllllllll}\mathrm{Cu} 1: & 1 & 2 & 3 & 4 & 5 & 6 & 7 & 8 & 9 & 10\end{array}$

Num $\quad 41230 \quad 58 \quad 94138190250 \quad 318 \quad 394$

Cum 5174710519933752777710951489

$\begin{array}{lllllllllll}\mathrm{Cu} 2: & 1 & 2 & 3 & 4 & 5 & 6 & 7 & 8 & 9 & 10\end{array}$

Num $\quad 41230 \quad 58 \quad 94138190250 \quad 318 \quad 394$

Cum 5174710519933752777710951489

$\mathrm{TD} 10=1489$

Vertex symbols for selected sublattice

Cu1 Point symbol: $\left\{6^{\wedge} 5.8\right\}$

Extended point symbol:[6.6.6.6.6(2).8(2)]

Cu2 Point symbol: $\left\{6^{\wedge} 5.8\right\}$

Extended point symbol:[6.6.6.6.6(2).8(2)]

Point symbol for net: $\left\{6^{\wedge} 5.8\right\}$

4-c net; uninodal net

Topological type: cds CdSO4; 4/6/t4; sqc5 (topos\&RCSR.ttd) $\left\{6^{\wedge} 5.8\right\}$ - VS [6.6.6.6.6(2).*] (72442 types in 11 databases)

Elapsed time: $29.54 \mathrm{sec}$. 


\section{Section 4: References}

[S1] Yang, G. W.; Zhang, Y. T.; Wu, Q.; Cao, M. J.; Wu, J.; Yue, Q. Y.; Li, Q. W. Q. Nitrogenrich 5-(4-pyridyl)tetrazole-2-acetic acid and its alkaline earth metal coordination polymers for potential energetic materials. Inorg. Chim. Acta. 2016,450, 364-371.

[S2] Zhu, Z.-K.; Lin, Y.-Y.; Yu, H.; Li, X.-X. Zheng, S.-T. Inorganic-Organic Hybrid Polyoxoniobates: Polyoxoniobate Metal Complex Cage and Cage Framework. Angew. Chem. Int. Ed. 2019, 58, 16864-16868.

[S3] Zhou, E.-L.; Qin, C.; Wang, X.-L.; Shao, K.-Z. Su, Z.-M. Steam-Assisted Synthesis of an Extra-Stable Polyoxometalate-Encapsulating Metal Azolate Framework: Applications in Reagent Purification and Proton Conduction. Chem. Eur. J. 2015, 21, 13058-13064.

[S4] Uchida, S.; Kawamoto, R.; Mizuno, N. Recognition of Small Polar Molecules with an Ionic Crystal of $\alpha$-Keggin-Type Polyoxometalate with a Macrocation. Inorg. Chem. 2006, 45, 51365144.

[S5] Zhou, E.-L.; Qin, C.; Huang, P.; Wang, X.-L.; Chen, W.-C.; Shao, K.-Z.; Su, Z.-M. A Stable Polyoxometalate-Pillared Metal-Organic Framework for Proton-Conducting and Colorimetric Biosensing. Chem. Eur. J. 2015, 21, 11894-11898.

[S6] Wei, M.; Chen, L.; Duan, X. A porous Cu(II)-MOF containing $\left[\mathrm{PW}_{12} \mathrm{O}_{40}\right]^{3-}$ and a large protonated water cluster: synthesis, structure, and proton conductivity. J. Coord. Chem. 2014, 67, 2809-2819.

[S7] Kawahara, R.; Uchida, S.; Mizuno, N. Redox-Induced Reversible Uptake-Release of Cations in Porous Ionic Crystals Based on Polyoxometalate: Cooperative Migration of Electrons with Alkali Metal Ions. Chem. Mater. 2015, 27, 2092-2099.

[S8] Li, Z.; Lin, L.-D.; Yu, H.; Li, X.-X. Zheng, S.-T. All-Inorganic Ionic Porous Material Based on Giant Spherical Polyoxometalates Containing Core-Shell $\mathrm{K}_{6} @ \mathrm{~K}_{36}-$ Water Cage. Angew. Chem. Int. Ed. 2018, 57, 15777-15781.

[S9] Wei, M.-L.; Sun, J.-J.; Duan, X.-Y. A Complex Based on a CuII-Schiff-Base Complex and POM-MOF Chain: Synthesis, Structure and Proton Conductivity. Eur. J. Inorg. Chem. 2014, 345-351.

[S10] Wei, M.; Wang, X.; Sun, J.; Duan, X. A 3D POM-MOF composite based on Ni(II) ion and 2,2'-bipyridyl-3,3'-dicarboxylic acid: Crystal structure and proton conductivity. J. Solid State Chem. 2013, 202, 200-206.

[S11] Hu, J.; Xu, Y.; Zhang, D.; Chen, B.; Lin, Z.; Hu, C. A Highly Symmetric Ionic Crystal Constructed by Polyoxoniobates and Cobalt Complexes for Preferential Water Uptake over Alcohols. Inorg. Chem. 2017, 56, 10844-10847.

[S12] Noro, S.; Tsunashima, R.; Kamiya, Y.; Uemura, K.; Kita, H.; Cronin, L.; Akutagawa, T.; Nakamura, T. Adsorption and Catalytic Properties of the Inner Nanospace of a Gigantic RingShaped Polyoxometalate Cluster. Angew. Chem. Int. Ed. 2009, 48, 8703-8706.

[S13] Miura, Y.; Imai, H.; Yokoi, T.; Tastumi, T.; Kamiya, Y. Microporous cesium salts of tetravalent Keggin-type polyoxotungstates $\mathrm{Cs}_{4}\left[\mathrm{SiW}_{12} \mathrm{O}_{40}\right], \mathrm{Cs}_{4}\left[\mathrm{PW}_{11} \mathrm{O}_{39}\left(\mathrm{Sn}-\mathrm{n}-\mathrm{C}_{4} \mathrm{H}_{9}\right)\right]$, and $\mathrm{Cs}_{4}\left[\mathrm{PW}_{11} \mathrm{O}_{39}(\mathrm{Sn}-\mathrm{OH})\right]$ and their adsorption properties. Microporous Mesoporous Mater. 2013, $174,34-43$. 
[S14] Uchida, S.; Eguchi, R.; Mizuno, N. Zeotype Organic-Inorganic Ionic Crystals: Facile Cation Exchange and Controllable Sorption Properties. Angew. Chem. Int. Ed. 2010, 49, 9930-9934.

[S15] Lesbani, A.; Kawamoto, R.; Uchida, S.; Mizuno, N. Control of Structures and Sorption Properties of Ionic Crystals of $\mathrm{A}_{2}\left[\mathrm{Cr}_{3} \mathrm{O}\left(\mathrm{OOCC}_{2} \mathrm{H}_{5}\right)_{6}\left(\mathrm{H}_{2} \mathrm{O}\right)_{3}\right]_{2}\left[\alpha-\mathrm{SiW}_{12} \mathrm{O}_{40}\right]\left(\mathrm{A}=\mathrm{Na}, \mathrm{K}, \mathrm{Rb}, \mathrm{NH}_{4}\right.$, Cs, TMA). Inorg. Chem. 2008, 47, 3349-3357.

[S16] Jiang, C.; Lesbani, A.; Kawamoto, R.; Uchida, S.; Mizuno, N. Channel-Selective Independent Sorption and Collection of Hydrophilic and Hydrophobic Molecules by $\mathrm{Cs}_{2}\left[\mathrm{Cr}_{3} \mathrm{O}\left(\mathrm{OOCC}_{2} \mathrm{H}_{5}\right)_{6}\left(\mathrm{H}_{2} \mathrm{O}\right)_{3}\right]_{2}\left[\alpha-\mathrm{SiW}_{12} \mathrm{O}_{40}\right]$ Ionic Crystal. J. Am. Chem. Soc. 2006, 128, 1424014241.

[S17] Ogasawara, Y.; Uchida, S.; Maruichi, T.; Ishikawa, R.; Shibata, N.; Ikuhara, Y.; Mizuno, N. Cubic Cesium Hydrogen Silicododecatungstate with Anisotropic Morphology and Polyoxometalate Vacancies Exhibiting Selective Water Sorption and Cation-Exchange Properties. Chem. Mater. 2013, 25, 905-911. 